\title{
An Exploratory Study Using Agent-Based Distillations to Investigate Reconnaissance Scenarios in Support of Higher Fidelity Combat Simulations
}

\author{
W. Chau and D. Grieger \\ Joint and Operations Analysis Division, Defence Science and Technology Group \\ Email: William.Chau@dsto.defence.gov.au
}

\begin{abstract}
Combat simulations and wargames form part of a suite of tools used to analyse complex military problems. The fidelity of this suite of tools varies in terms of their representation of both physical parameters and military doctrine. Higher fidelity models often have associated resource and computational constraints which makes exploring large regions of the parameter space infeasible. An approach known as operational synthesis employs low fidelity agent based distillation (ABD) models to rapidly search this parameter space in order to identify areas of interest for further exploration in higher fidelity models.

In this study we use the Map Aware Non-Uniform Automata (MANA) ABD model to gather additional insights for a reconnaissance study that was conducted in a higher fidelity combat simulation known as COMBATXXI (CXXI). The CXXI study investigated the potential impact of variations to platform mobility, sensors and signatures on the effectiveness of land combat vehicles conducting an observation task against an airfield and a river crossing point. An existing MANA scenario was used to explore the impact of factors not considered in the CXXI study, specifically, variations to both observation post placements and schemes of manoeuvre.
\end{abstract}

The MANA model identified the same trends in relation to mobility and signature that were identified in the CXXI study. The results from the additional variations performed in the MANA model showed a significant difference in the number of observations when a more aggressive and direct scheme of manoeuvre was employed and where alternative observation post locations were used. We explore these results further by implementing them in CXXI in order to provide greater confidence in the findings and further insights into the magnitude of the differences.

Keywords: $\quad$ Agent-based modelling, Defence, simulation, operations analysis 
Chau and Grieger, An Exploratory Study using Agent-Based Distillation to Investigate Reconnaissance Scenarios in Support of Higher Fidelity Combat Simulations

\section{INTRODUCTION}

Analytical combat simulations are often employed as part of a suite of tools used to explore complex military problems (Bowley, 2003). Supported by methods such as analytical wargaming and historical studies these simulations can help investigate the cause-and-effect relationships in a complex military environment (GUIDEx, 2006). Many high fidelity analytical combat simulations models have associated resource and computational constraints which limits exploring large regions of the parameter space. In these cases it may be desirable to explore the parameter space using a lower fidelity model in order to identify points of interest that can be fed back into a higher fidelity model for further analysis.

Operational Synthesis is an analysis technique developed by the US Marine Corps Combat Development Command to integrate a range of military simulation and decision support techniques to help address the limitations of each individual tool (Horne, 2001). Operational Synthesis is different from other multi-method techniques in that it integrates Agent Based Distillations (ABDs) into the suite of modelling tools. As the name implies, ABDs deliberately distil specific modelling variables in order to focus on those relevant to the study in question. These properties of ABDs allow for multiple parameters to be explored in a shorter period of time than is possible with analytical wargaming and other high-fidelity combat simulations. In previous work, Anderson et al. (2003) found that an ABD produced "reasonable match' for results from an analytical wargaming tool.

In a previous paper we showed that the Map Aware Non-Uniform Automata (MANA) ABD (McIntosh et el, 2007) was able to produce trends consistent with a higher fidelity combat simulation in the context of small combat teams conducting close combat in an urban setting (Chau and Grieger, 2013). In this paper we use MANA to explore additional parameters in a reconnaissance study that was conducted using the COMBATXXI (CXXI) analytical simulation ${ }^{2}$. The CXXI study explored the impact of variations to platform mobility and signatures on the effectiveness of land combat vehicles conducting an observation task against an airfield and a river crossing point. In addition to mobility and sensor variations we also explore changes made to the opposing red force scheme of manoeuvre (SoM) and the observation post (OP) locations for the friendly blue force in the MANA ABD. We identify which combinations of these additional parameters warrant further exploration and explore their effect in more detail by running additional scenarios in the higher fidelity CXXI model.

\section{METHOD}

A reconnaissance scenario previously developed by DSTO (internal Defence Study) in MANA was used for this study. It was chosen because it represented a good abstraction of the terrain, context and mission of the CXXI scenario. The MANA scenario consisted of a red force traversing through terrain and a blue force representing four OPs. The red forces started at the same initial positions before travelling two separate SoMs to finish at the same final waypoint. The SoMs included river crossing and dense terrain for concealment. The blue forces were located in static positions where they were able to observe possible routes of the red force. Blue forces were employed with a maximum sensor of $3000 \mathrm{~m}$ which was adequate enough to cover most (not all) of the battlespace.

The most significant difference in the fidelity of the MANA model when compared to CXXI is in relation to the terrain. MANA represents elevation as a step model rather than CXXI's high-resolution elevation databases. The vegetation in MANA was represented by its light and dense bush models. The light bush model reduces an entity's movement speed by a factor of 25 per cent and reduces its probability of detection by a factor of 30 per cent. The dense bush model reduces an entity's movement speed by 80 per cent and reduces its probability of detection by 90 per cent. In CXXI vegetation is modelled in greater fidelity and using more complex algorithms to determine the effect on movement and acquisition.

In the MANA scenario, the vehicle speed and signatures for the red force were varied to confirm that the scenario was providing intuitive results; adding confidence for further analysis. An important component of any ABD study is to ensure that the level of abstraction of the distilled model still produces plausible results; otherwise there would be little point continuing the study.

\footnotetext{
${ }^{1}$ This is an internal Defence study

${ }^{2}$ COMBATXXI (Combined Arms Analysis Tool for the 21st Century) is a United States Joint Army-Marine Corps effort that replaced the Combined Arms and Support Task Force Evaluation Model (CASTFOREM) in 2009
} 
Chau and Grieger, An Exploratory Study using Agent-Based Distillation to Investigate Reconnaissance Scenarios in Support of Higher Fidelity Combat Simulations

Vehicle speed was modified by factors of two, three and four while the chance of being detected was reduced by 30 per cent, 60 percent and 90 per cent. Once the results for the speed and signature parameters were assessed for plausibility, SoM were varied for the red force and the locations of the OPs were varied for the blue force (Table 1). These parameters were chosen as the authors noticed sensitivities around the locations of the entities in the CXXI study. These variations to each of the four parameters resulted in a total of 256 scenario combinations each of which was replicated 100 times in MANA to support statistical analysis of the outcomes. From these results we identified the variables that had a significant effect on the total number of observations made by the blue force on the opposing red force. This was also the metric used in the CXXI study.

The analysis of the MANA results was then used as a driver for identifying three additional CXXI scenario alternatives which were then developed, run and analysed in CXXI.

Table 1. Variable definitions for data farming.

\begin{tabular}{|c|c|c|c|}
\hline Force & Variable & Naming Convention & Description \\
\hline \multirow{4}{*}{ Blue } & \multirow{4}{*}{$\begin{array}{l}\text { Number and } \\
\text { location of } \\
\text { observation } \\
\text { posts }\end{array}$} & OP-Baseline & $\begin{array}{l}\text { A baseline observation post location based from a previous MANA } \\
\text { scenario. }\end{array}$ \\
\hline & & OP-Plus & $\begin{array}{l}\text { Two additional observation posts were added and one re-located to } \\
\text { close proximity of the final waypoint. }\end{array}$ \\
\hline & & OP-Minus & One observation post was removed from the baseline. \\
\hline & & OP-Relocation & $\begin{array}{l}\text { No observation posts were removed. However, two observation } \\
\text { posts were relocated based on military SME advice. }\end{array}$ \\
\hline \multirow{4}{*}{ Red } & \multirow{4}{*}{$\begin{array}{l}\text { Scheme of } \\
\text { manoeuvre }\end{array}$} & SoM-Baseline & $\begin{array}{l}\text { A baseline scheme of manoeuvre based from a previous MANA } \\
\text { scenario. }\end{array}$ \\
\hline & & SoM-Indirect & A less-direct route being taken by the red force. \\
\hline & & SoM-Direct & A more-direct route being taken by the red force. \\
\hline & & SoM-Dynamic & $\begin{array}{l}\text { A dynamic scheme of manoeuvre taken by the red force. The } \\
\text { entities were given additional characteristic of being attracted to } \\
\text { concealment and repulsed by observation posts. }\end{array}$ \\
\hline
\end{tabular}

\section{RESULTS}

The results below are presented in two sections. The first section analyses the various combinations of parameters in the MANA scenario while the second section analyses the results from the additional vignettes that were implemented into CXXI.

\subsection{MANA results and data farming insights}

The MANA results showed that the number of observations of the Red force vehicles by blue force decreased as both the speed of the Red vehicles increased and as the Red signature (chance of observation per attempt) decreased (Figure 1). These trends were also identified in the CXXI study. While these results are intuitive, they are important because they provide valid and plausible findings that justify further parameter exploration in MANA.

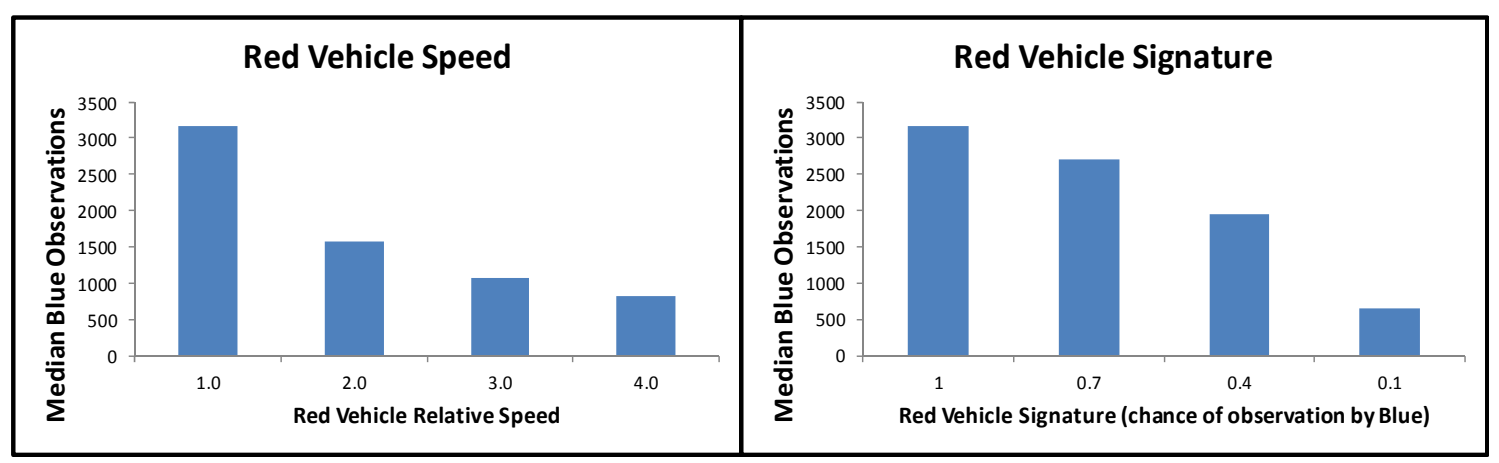

Figure 1. Red vehicle speed and Red signature MANA results. 
Chau and Grieger, An Exploratory Study using Agent-Based Distillation to Investigate Reconnaissance Scenarios in Support of Higher Fidelity Combat Simulations

The results from the MANA simulation of alternative blue OP locations and red force SoM produced both normal and non-normal distributions ${ }^{3}$. Hence, a Kruskal-Wallis ${ }^{4}$ test was used to compare the median number of observations of red vehicles made by blue forces. These comparisons were made for each of the 16 possible vignette combinations of OP placement and red force SoM using the baseline vehicle speed and signature. We rank both the performance of each SoM (Table 2) and each OP (Table 3). Each cell in the table represents a statistically significant difference from the other options within that column. For example, in Table 3 for SoM-Baseline we see that OP-Plus resulted in statistically fewer observations than the other OP options and that there was no statistical difference in the number of observations for OP-Baseline and OPRelocation.

Table 2. MANA observation SoM performance rankings.

\begin{tabular}{|c|c|c|c|c|}
\cline { 2 - 5 } \multicolumn{1}{c|}{} & OP-Baseline & OP-Plus & OP-Minus & OP-Relocation \\
\hline $\begin{array}{c}\text { Least } \\
\text { observations } \\
\downarrow\end{array}$ & SoM-Direct & SoM-Direct & SoM-Direct & SoM-Direct \\
\cline { 2 - 5 }$\downarrow$ & SoM-Dynamic & SoM-Dynamic & SoM-Dynamic & SoM-Dynamic \\
\cline { 2 - 5 } $\begin{array}{c}\text { Most } \\
\text { observations }\end{array}$ & SoM-Indirect & SoM-Indirect & SoM- Baseline & SoM- Baseline \\
\cline { 2 - 5 } & SoM-Baseline & SoM- Baseline & SoM-Indirect & SoM-Indirect \\
\hline
\end{tabular}

The more direct red force SoM produced the most favourable outcome for the red force regardless of the location of blue OPs. This result intuitively implies that the terrain covered in the longer baseline SoM did not provide the additional level of concealment required to justify the additional distance covered. Given the limitations of the terrain model in MANA the authors identified this result as something that should be explored further using a higher fidelity model.

Table 3. MANA observation OP location performance rankings.

\begin{tabular}{|c|c|c|c|c|}
\cline { 2 - 5 } \multicolumn{1}{c|}{} & SoM-Baseline & SoM-Indirect & SoM-Direct & SoM-Dynamic \\
\hline $\begin{array}{c}\text { Most } \\
\downarrow\end{array}$ & OP-Plus & OP-Relocation & OP-Relocation & OP-Relocation \\
\cline { 2 - 5 }$\downarrow$ & $\begin{array}{c}\text { OP-Baseline/ } \\
\text { OP-Relocation }\end{array}$ & OP-Plus & OP-Plus & OP-Plus \\
\cline { 2 - 5 } $\begin{array}{c}\text { Least } \\
\text { observations }\end{array}$ & OP-Minus & OP-Minus & OP-Minus & OP-Minus \\
\cline { 2 - 5 }
\end{tabular}

The MANA model also showed that the various OP locations had a significant impact on the number of observations made. In particular, OP-Relocation produced statistically more observations relative to the indirect, direct and dynamic SoMs even though OP-Plus had additional OPs. A slightly different result was observed for the baseline SoM where there was a benefit in having additional OPs. These two insights once again imply an intuitive result in that the coverage provided by all OPs is the critical component of OP selection.

The results from MANA in relation to OP placement and red force SoM warrant further investigation in CXXI to provide additional evidence to support the preliminary findings. As such three additional vignettes were created in CXXI to compare with the existing reconnaissance study results as described below:

(i) More direct Red SoM

(ii) OP-Relocation

(iii) OP-Relocation and direct Red SoM.

\footnotetext{
${ }^{3}$ A Shaprio-Wilk normality test with an alpha value of 0.05 was used.

${ }^{4}$ Tests were conducted using Breakpoint Analysis with Nonparametric Data Option (BRANDO) software (Emond and Turnbull, 2006)
} 
Chau and Grieger, An Exploratory Study using Agent-Based Distillation to Investigate Reconnaissance Scenarios in Support of Higher Fidelity Combat Simulations

\subsection{CXXI results and insights}

CXXI has a higher fidelity observation model than MANA and provides four levels of acquisition: detection, classification, recognition and identification. In order to compare our MANA insights with the CXXI results we initially focus on the total number of observations (Figure 2). The results from the additional three vignettes described in the previous section also displayed non-normal distributions. The CXXI results indicated that there were statistical differences in the number of total observations from some of the additional vignettes (Figure 2). In contrast to the MANA study, the more direct SoM did not result in fewer observations of red force when compared to the baseline. The more

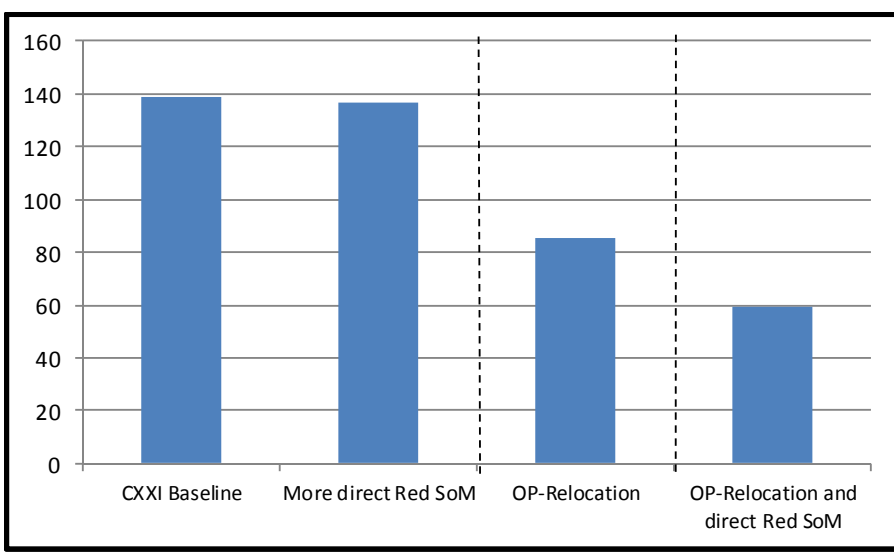

Figure 2. CXXI results: Median number of total observations. Dashed vertical lines separate results that are statistically significant.

direct red force SoM did however produce statistically fewer observations when the OP-Relocation locations were used by the blue force. In order to understand these results in more detail a heatmap showing the locations and frequency of each detection was produced (Figure 3). The red shadings indicate the areas where the most detections occurred while the transition to green indicates decreasing frequencies.

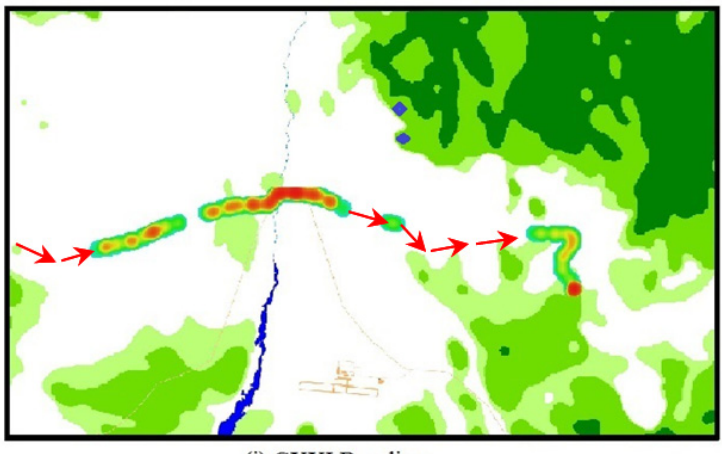

(i) CXXI Baseline

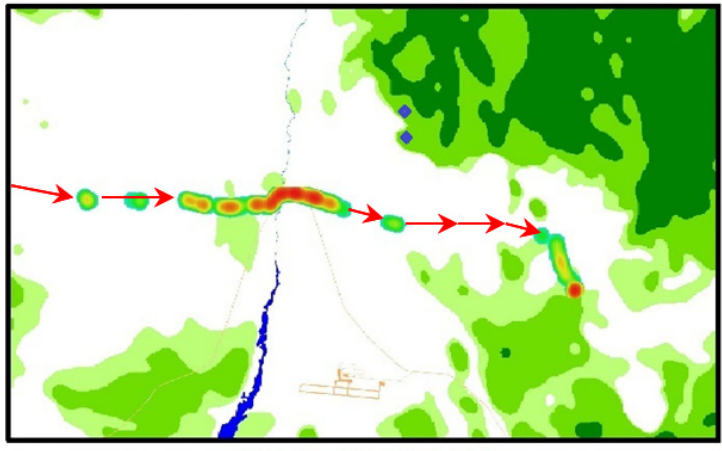

(iii) More direct Red SoM

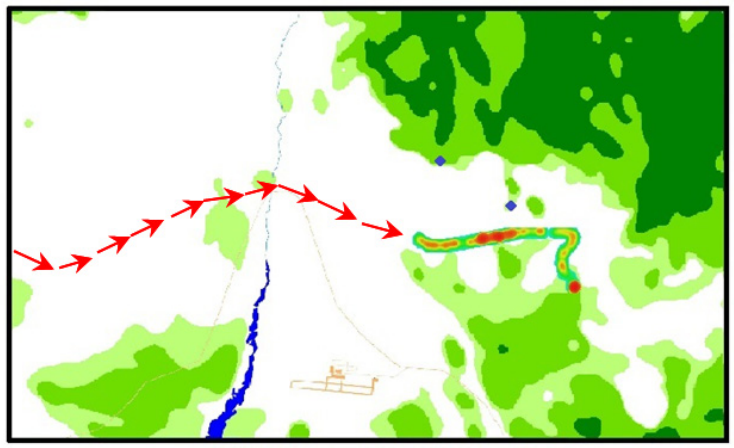

(ii) Alternative Blue OP Locations

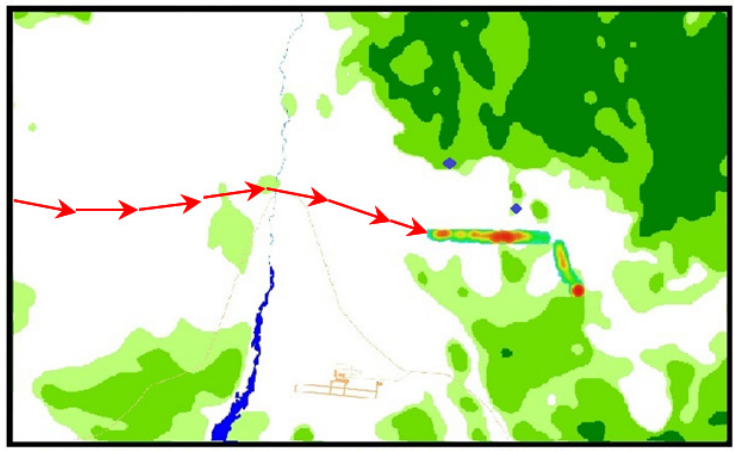

(iv) Alternative Blue OP locations and direct Red SoM

Figure 3. CXXI results: Heat maps of observations

Despite there being no statistical difference in the number of detections between the baseline red force SoM and the more direct alternative, there appears to be fewer detections during the earlier stages of the vignette (Figure 3 (i) and (iii)). A visual inspection of the CXXI terrain in these areas indicates that a difference in elevation between the two SoMs was the likely cause of these differences. Further analysis is required to determine the statistical significance of these results and to determine what the corresponding effect might be in a combat environment as these differences could be crucial to the mission of blue force depending on its mission. 
Chau and Grieger, An Exploratory Study using Agent-Based Distillation to Investigate Reconnaissance Scenarios in Support of Higher Fidelity Combat Simulations

The blue force was unable to make any observations of red during the earlier stages of the vignette when using the OP-Relocation. However, a more detailed analysis of the CXXI data ${ }^{5}$ revealed that the OPRelocation produced far more instances where an observation level of 'Recognition' was achieved. These differences could be crucial to the outcome in a combat environment due to better quality observations resulting in more actionable events.

\section{SUMMARY}

In this study, we have demonstrated the ability of ABDs to be integrated with higher fidelity models to support the analysis of a reconnaissance scenario. The MANA model produced intuitive results in that the number of observations of opposing force land combat vehicles decreased as the speed of the vehicle increased or the signature decreased. More significantly the MANA model identified that the total number of observations was sensitive to the opposing force SoM and the location of friendly force observation posts.

Based on the findings from the MANA model three additional CXXI scenarios were constructed to explore the effect of enemy SoM and friendly OP location in more detail. The alternative enemy SoM resulted in fewer observations earlier in the scenario while the OP-Relocation resulted in significantly fewer observations earlier in the scenario but significantly more toward the latter stages of the SoM. While engagements were not modelled in this scenario it is likely that these observation numbers would have a flow on effect to other battlefield effects if additional activities were modelled.

Even though these results are somewhat intuitive it is important to note that the true value of the MANA model in this study was in its ability to inform the scenario reduction process. This process allowed potential sensitivities within the CXXI study to be identified and explored without requiring the significant resource overhead that would normally be required to explore such a large space entirely within CXXI. It is possible however that as distributed computing capabilities increase the potential for MANA and other ABDs to contribute in the manner described in this paper may reduce. In addition, because by definition military ABDs have deliberately low fidelity terrain, observation and engagement models, their results should always be used to inform further studies rather than to provide robust conclusions.

\section{REFERENCES}

Anderson, M.A., Lauren, M.K. and Galligan, D.P. (2003). Exploring the 2002 Seminar Games Janus Wargaming results with MANA, Defence Technology Agency New Zealand, DTA-R-196

Bowley D., Castles T. and Ryan A. (2003). Constructing a SUITE of Analytical Tools: A Case Study of Military Experimentation, ASOR Bulletin 22(4): 2-10.

Chau, W. and Grieger, D. (2013). Operational synthesis for small combat teams: exploring the scenario parameter space using agent-based models. In Gaertner, P., Bowden, F., Piantadosi, J. and Mobbs, K. (eds) 22nd National Conference of the Australian Society for Operations Research (ASOR 2013). The Australian Society for Operations Research, Adelaide, December 2013, pp. 196-203. http://www.asor.org.au/conferences/asor2013/J7/chau.pdf

Emond, E.J. and Turnbull, A.E. (2006) BRANDO - Breakpoint Analysis with Nonparametric Data Option, DRDC-CORA-TM-2006-40, Centre for Operations Research, Canada

Horne, G. E. (2001). Beyond Point Estimates: Operational Synthesis and Data Farming, Maoeuvre Warfare Science 2001, Quantico VA.

McIntosh, G.C., Galligan, D.P., Anderson, M.A. and Lauren, M.K. (2007). MANA (Map Aware Non-uniform Automata) Version 4 User Manual. DTA Technical Note NR-1465, New Zealand: Defence Technology Agency

TTCP (2006), Guide for Understanding and Implementing Defense Experimentation (GUIDEx). Washington DC, The Technical Cooperation Program (TTCP), Joint Systems Analysis (JSA) Group, Methods and Approaches for Warfighting Experimentation Action Group 12 (AG-12)

\footnotetext{
${ }^{5}$ CXXI has four levels of observation within its data: detection, classification, recognition and identification. The observation levels differ depending on sensors and range of entities.
} 